Mihael Gudlin

Miro Hegedić

Hrvoje Cajner

Nedeljko Śtefanić

https://doi.org/10.21278/TOF.43201

ISSN 1333-1124

eISSN 1849-1391

\title{
SOLVING PROBLEMS OF INTERRUPTIONS AND MULTITASKING IN THE PHARMACY OF A LARGE HOSPITAL CENTRE
}

\begin{abstract}
Summary
This paper presents an approach to solving problems of interruptions and multitasking in inpatient pharmacy processes of a large hospital centre, which is based on statistical modelling and simulations. The approach is applied to the process of receiving deliveries (from suppliers) to determine the feasibility of improvements in the organization of work. In the initial phase of research, data on the deliveries from suppliers were collected during the time study and a typical daily load on the pharmacy staff and infrastructure in the current state was simulated. Subsequently, a new organizational model, which included two defined blocks of time for delivery, was suggested and three simulation scenarios were created to examine the effects of new organization of work on daily activities. Finally, a comparison of system constraints and results obtained by the simulation models confirmed the feasibility of the proposed improvements. By implementing the new organization of work, it will be possible to avoid overlapping in pharmacy processes, which will reduce interruptions to work and the need for multitasking and will finally result in fewer errors in work.
\end{abstract}

Key words: $\quad$ inpatient pharmacy, process improvement, statistical modelling and simulation, supplier arrivals, goods receiving process

\section{Introduction}

From the organizational and infrastructural perspective, hospitals are complex systems whose quality of services and efficiency depend on the interaction between various processes. Some of the main processes in a hospital are logistics processes which are complex [1] and have a significant impact on costs. With their contribution of up to $30 \%$ in the costs of a hospital [2], they represent the second highest costs, immediately after the cost of hospital staff salaries. There is the potential to cut this cost in half by managing the logistics processes well. The main part of the hospital logistics processes is a hospital pharmacy contributing up to $80 \%$ in the total hospital logistics cost [3], which also has an impact on the service quality of other hospital departments (e.g. waiting time for medication distribution) [4]. Although a pharmacy has a significant role in the operational efficiency of the hospital, there are still problems with measuring its efficiency [5]. Activities and processes of the hospital pharmacy could be divided into two types of services: distributive and clinical [5]. The main activities of 
the hospital pharmacy include procurement, storage, and distribution of goods and dose preparation for the dispensation of medications [6]. In this paper, the process of receiving deliveries from a supplier in the inpatient pharmacy of a large hospital centre has been explored. In the observed pharmacy, certain problems were detected, such as errors in the receiving and issuing of goods, interruptions to work, and a constant need for multitasking, which was perceived by employees as an increase in their workload. These problems were attributed to the current organization of work that caused overlapping in the pharmacy processes.

\subsection{Literature review}

To stress the importance of the researched subject, we have done a literature review to learn how these types of problems in a pharmacy are dealt with and to identify approaches used to improve pharmacy processes. We organized papers based on the afore-mentioned problems in a pharmacy into three main topics: 1) effect of medication-related errors, 2) effect of work interruptions, and 3) effect of workload on employees.

When considering the impact of an inpatient pharmacy on the hospital, it is important to mention that Wittich et al. [7] recognized medication errors and errors related to the process of administration and management of medication as important causes of death of patients (1 of 854 inpatient deaths is connected to medication errors). Keers et al. [8] found through their literature review that a pharmacy contributes to medication errors through delayed deliveries, incorrect dispensation of medication and unavailability of the required medication in stock. Behzad et al. [9] used the USA healthcare system as an example to show the importance of medication errors and mentioned that medication-related errors cost the nation at least $\$ 3.5$ billion per year. They also believe that improvements in the pharmacy processes can reduce these costs.

Interruptions and distractions at work are recognized by pharmaceutical technicians as factors that have the biggest impact on the medication preparation errors in a pharmacy [10]. These factors also prolong the time required for standard activities done by pharmacists and pharmaceutical technicians by $27 \%$ on average [11]. Additionally, results in [12] indicated that errors during the dispensation of medication are related to the process of organization of work, system for dispensation, work interruptions, and staff workload. The conclusion made by Lea et al. [13] is that interruptions, multitasking, and task-switching could be minimized by changes in the organization of work.

There is evidence that heavy workload affects the quality of services provided by pharmacists. It also affects their physical and mental condition [14]. Research findings in [15] showed that the main factors influencing the stress of pharmacy employees are insufficient number of employees(heavy workload), frequent breaks and interruptions in work, and the need to work with third parties (e.g. suppliers). The consequences of stress, on the other hand, are work dissatisfaction, absenteeism, and workforce fluctuation.

Different approaches to pharmacy process improvement have been found in literature, including implementation of automation [16], simulations and mathematical optimization [17-26], and improvement methodologies [27-29]. James et al. [16] investigated the influence of automation on reducing errors in dispensation of medication. The authors showed that automation increases productivity by $43 \%$, but that it also reduces dispensation errors by $56 \%$, although other types of errors occur (e.g. labelling errors).

Lu et al. [17] investigated a hospital pharmacy at a Community hospital and simulated the process of delivery of a medications ordered by the hospital. Results obtained from the simulation model were used as a recommendation to improve the delivery of medications 
ordered by the hospital. The results implied a possible reduction in the delivery time by $20 \%$. Hong Choon et al. [18] used simulation models to investigate connections between different scheduling strategies in the outpatient pharmacy and the patient waiting time. Based on simulation model results, Greenroyd et al. [19] recommended a new layout of a pharmacy that should result in a reduction in the walking time of the pharmacy employees by $24 \%$. Day et al. [20] proposed an appropriate staffing level in a pharmacy based on the simulation results with the goal of reducing the patient waiting time. They concluded that it is possible to reduce the waiting time to only 30 seconds by increasing the number of employees responsible for the process from 2 to 6 . Bahadori et al. [21] used queuing theory to assess the current state of outpatient pharmacy processes. Subsequently, a simulation model was used to define the appropriate staffing level in the process of delivery. It is interesting to notice that they are talking about the benefits of multitasking and task switching, although only theoretically. Using the simulation model with 18 different scenarios, Spry and Lawley [22] investigated the impact of the number of employees and scheduling on the average time of pharmacy medication delivery. Sawik [24-26] did extensive research regarding the allocation of personnel to supporting services in a hospital, including pharmacy services. He used multicriteria mathematical optimization with the objective of minimizing operating costs of supporting services and maximizing the total number of employees in the case of a biobjective model. In the case of a triple-objective model, the defined objective was the minimization of operating costs of supporting services and the maximization of the total number of employees and of the total number of permanent employees. Results of the research showed that the number of hired workers can be reduced in almost all departments of the hospital.

Jenkins and Eckel [27] used the principles of lean methodology and work and time studies to develop two models of possible improvement in the work processes of pharmacy employees. By removing activities that do not add value, the first model was able to decrease the non-value-added time by $55 \%$ and the other by $74 \%$. In the study [28], the authors discovered that it is possible to shorten the cycle of medication delivery process by more than $45 \%$ by applying the DMAIC approach and the $5 \mathrm{~S}$ lean tool. Sullivan et al. [29] reported that it is possible to shorten the pharmacy medication delivery time by applying the value stream mapping and by reducing unnecessary steps in the process.

\subsection{Research purpose and objectives}

The literature review has revealed several important gaps. Firstly, research on logistics activities within hospitals has not got priority in literature [30], and this is particularly true of the logistics processes of the hospital pharmacy that still have not been sufficiently researched. Furthermore, the hospital external distribution and scheduling are hardly covered in literature [30]. Secondly, there are only a few papers that deal with improvements in pharmacy processes, regarding the reduction in the number of interruptions and in multitasking brought about by changes in the organization of work [13]. Analysis of the roots of errors in pharmacies is often focused just on the activities of an individual and not on the system and processes that lead to these errors [31]. Finally, there is still an open question about what to do when there are no opportunities for investment in automation, infrastructural changes, or new employees, and there is no structured data on the current state of the process so that improvements can be made immediately.

The objective of this paper is to contribute to the research area by demonstrating an approach to the problems of interruptions and multitasking in the processes of the inpatient pharmacy of a large hospital centre. The approach, based on statistical modelling and simulation, solves these problems through changes in the organization of work. We used this 
approach for determining the potential for improvement and the feasibility of implementing the new organization of work in the pharmacy, using the concrete example of the process of receiving deliveries. Our hypothesis was that such an approach was appropriate to the conditions in which no structured data of the current state of the process were available and the project had constraints regarding investments in new resources.

In the research into the process of receiving a delivery and into the applicability of the new organization of work, we were guided by five questions:

- Q1: Is there a significant variation in the number of supplier arrivals within the observed days?

- Q2: Is it possible to group deliveries using the information about the delivery processing time?

- Q3: How much time is used in a typical workday for the process of receiving deliveries using the available resources (employees, unloading positions, time)?

- Q4: What is the impact of different distributions of the supplier arrivals in defined blocks of time on the process of receiving deliveries on a daily basis?

- Q5: Is it possible to define blocks of time for receiving deliveries and still receive all deliveries on a daily basis?

\section{Research setting}

The pharmacy whose processes were researched is part of the largest hospital centre in the Republic of Croatia with 33 clinics, 1975 beds, and about a million medical cases, among which 72000 were inpatient medical cases according to the data from 2014. With further expansion of the hospital planned (building new clinic departments), the number of inpatient medical cases is expected to increase. This trend will consequently lead to an increase in the demand for services from the inpatient hospital pharmacy, such as delivery of medications and medical consumables to the clinics, but also to an increase in the number of supplier arrivals and deliveries. Investment in the construction of new clinics limits the ability to invest in new equipment and to hire new employees for the hospital pharmacy. The aforementioned facts have served as motivation to hospital management to initiate a performance improvement project related to the hospital pharmacy.

In September 2015, the initial meeting was held and a team responsible for the pharmacy project realization was formed. The project team included a team leader, a project manager, pharmacy employees, and external consultants. The scope of the project was limited to the processes of the pharmacy, with planned project duration of 3 months. The two main project objectives were improvement in the delivery lead time [32] and improvement in the process of receiving deliveries from suppliers. In this paper, the focus is on the latter objective. The resources of the project were limited to the time that the staff can invest in the project.

The investigated inpatient pharmacy is located in a building that was not initially designed for a pharmacy but was later adapted to suit its requirements. The assortment of the pharmacy consisting of approximately 1600 items has been divided into several areas. In the reception area, the pharmacy has three available positions for receiving deliveries. The infrastructure conditions and technological capacities of the pharmacy are not good, with a high volume of manual work in daily routines and processes. The current organization of work and infrastructural constraints of the pharmacy workspace result in physical overlapping of process flows of receiving deliveries and issuing medications and other ordered items to clinics, as shown in Figure 1. 


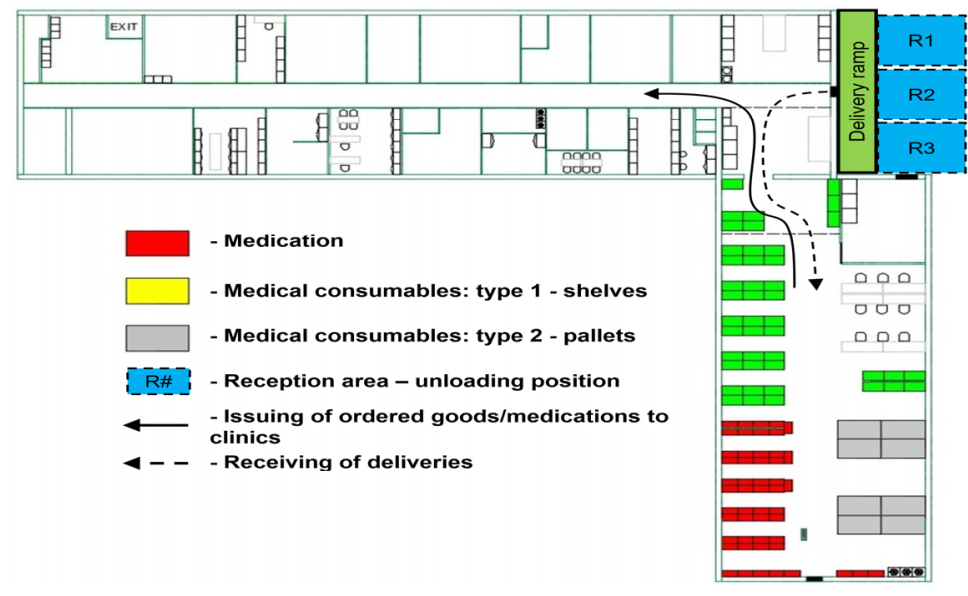

Fig. 1 Pharmacy layout

During the project, thirty-three employees were employed in the central hospital pharmacy, nine pharmacists, eighteen technicians, and six members of support staff. The pharmacy works around the clock, with the basic eight-hour shift from 8:00-16:00, and the rest of the time the pharmacy is on call. Deliveries are received and stored only during the basic shift. Two pharmacists and three technicians are charged with the tasks of issuing and storing medications at any time. The warehouse for medical consumables is staffed with two pharmacists, three technicians, and three support employees at any time.

\section{Methodology}

The selected research approach consists of four phases: 1. collection of data by carrying out the time study, 2. analysis of data and statistical modelling, 3. development of a simulation model of the current state of the process under study, and 4. evaluation of the process improvements obtained by new simulation models.

\subsection{Time study}

The goal of this step in the research was to collect relevant data about the supplier arrivals and the process of receiving deliveries. Thus, we developed a time study form which included the information about the date of delivery, the name of the supplier, the type of goods delivered, the time of arrival of goods and the time of departure from the reception area, as shown in Table 1. In order for the time study sample to be a good representation of monthly supplier arrivals and deliveries, we decided to collect data during five working days: two days at the beginning of the month, two in the middle, and one at the end of the month. The data was recorded in a time period from 8 a.m. to 2 p.m., a decision that was based on our agreement with the pharmacy employees who claimed that deliveries after 2 p.m. are highly unlikely.

Table 1 Time study form [33]

\begin{tabular}{|l|l|l|c|c|c|c|}
\hline \# & $\begin{array}{l}\text { Name of } \\
\text { supplier }\end{array}$ & $\begin{array}{l}\text { Type of goods } \\
\text { delivered }\end{array}$ & $\begin{array}{l}\text { Date of } \\
\text { delivery }\end{array}$ & $\begin{array}{c}\text { Time of } \\
\text { arrival } \\
\text { [h:min] }\end{array}$ & $\begin{array}{c}\text { Time of } \\
\text { departure } \\
\boldsymbol{t}_{\mathbf{d}} \\
{[\mathbf{h : m i n}]}\end{array}$ & $\begin{array}{c}\text { Delivery } \\
\text { processing time } \\
\boldsymbol{t}_{\text {pro }}=\boldsymbol{t}_{\mathbf{d}}-\boldsymbol{t}_{\mathbf{a}} \\
{[\mathbf{h : m i n}]}\end{array}$ \\
\hline 1. & Supp1 & Medications & Day1 & $11: 00$ & $11: 04$ & $0: 04$ \\
\hline 2. & Supp1 & Medications & Day2 & $10: 49$ & $10: 51$ & $0: 02$ \\
\hline 3. & Supp2 & Medical consum. & Day1 & $10: 22$ & $10: 43$ & $0: 21$ \\
\hline 4. & Supp2 & Medical consum. & Day2 & $10: 07$ & $10: 09$ & $0: 02$ \\
\hline
\end{tabular}

Types of goods delivered were divided into three groups: medications, medical consumables, and mixed deliveries. We calculated the delivery processing time as the 
difference between the arrival time, i.e. the moment the supplier parked his vehicle in one of the three available positions of the reception area and the time he left the reception area. The activities included in the delivery processing time are presented in Figure 2.

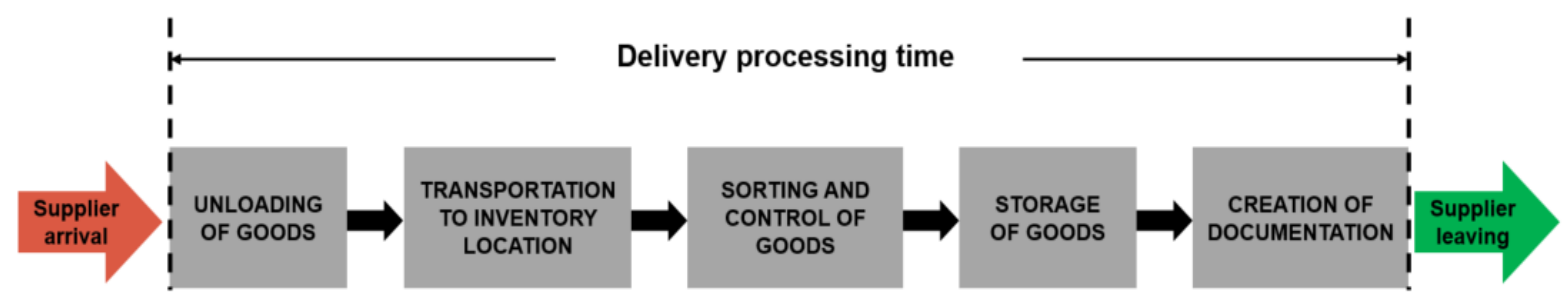

Fig. 2 Activities included in the process of receiving a delivery [33]

\subsection{Data analysis and statistical modelling}

From the data obtained through the time study, we calculated the sample statistics regarding the number of supplier arrivals during the observed days. A probability distribution of supplier arrivals was estimated and tested via the $\chi^{2}$ goodness of fit test. Information regarding the processing times necessary for receiving deliveries was used to make an initial analysis of deliveries and their clustering in separate groups. The clustering was carried out by the k-means method [34,35]. We varied the centre parameter of the k-means algorithm between values of 2, 3 and 4 while setting the maximum number of iterations to 20 . The algorithm was started 20 times for each selected value of the centre parameter with the purpose of ensuring a stable solution; i.e., to see if we get approximately the same cluster structure and centres. Differences in the delivery processing times of formed groups obtained by the k-means method were formally tested by Mood's median test. This type of test was used because the recorded data did not correspond to the normal distribution, the variances across the groups were not equal, and a number of outliers were detected. Finally, we fitted the theoretical probability distributions to every delivery group, using parameters of group centres. The fitted distributions to distinct groups were also tested by the $\chi^{2}$ test.

\subsection{Simulation model of the current state}

The Monte Carlo method [36] was chosen for the development of a simulation model of the observed process (scenario $0-\mathrm{S} 0$ ). The reasoning behind this decision was based on our need to obtain estimates of operating characteristic of the process in a short time span using the limited amount of data we gathered. The idea was to simulate supplier arrivals and the processing times required for receiving delivered goods that occur in a typical working day in the pharmacy. To get good estimates of population parameters, 10000 iterations were conducted during the simulation process.

Simulation process was divided into four stages:

- Stage 1: The number of supplier arrivals per day was simulated by using distribution parameters of supplier arrivals. An expected number of supplier arrivals per day were estimated as the average of supplier arrivals of all simulation iterations. The confidence interval of supplier arrivals at the level of significance of 5\% was determined by taking 2.5 percentile and 97.5 percentile of all simulation iterations of supplier arrivals.

- Stage 2: A simulation of types of deliveries per day was done using the information about the proportion of formed groups of deliveries obtained by the k-means algorithm. The simulated delivery types were then passed to supplier arrivals from stage 1 .

- Stage 3: Distribution parameters of processing times for different types of deliveries were used to simulate the processing times of deliveries simulated in stage 2 . 
- Stage 4: The total processing time of single iterations was calculated. The median value of all simulated total processing times was taken to represent the typical processing time per day, and interquartile range of processing times of iterations was used to get a range of plausible values of processing time.

\subsection{Evaluation of process improvements}

In the last phase of the research, the feasibility of daily pharmacy activities related to the arrival of suppliers and the process of receiving deliveries was examined in the case of defining two distinct blocks of time that were reserved only for this type of activity. We created three new simulation scenarios with common predefined two-hour blocks, the first block in the period from 8 a.m.- to 10 a.m. and the second from 12 p.m.to 2 p.m.

Since four hours are available in the proposed new organization of the process of receiving deliveries in the studied hospital pharmacy, as opposed to six hours available in the existing organization of work, we have decided to check possible distributions of supplier arrivals and total delivery processing times by using the afore-mentioned simulation scenarios, which have the following properties:

- Scenario 1: All suppliers who came during the period from 10 a.m.to 2 p.m. were assigned to the second block of time and the rest were assigned to the first;

- Scenario 2: All suppliers who came during the period from 8 a.m. to 12 p.m. were assigned to the first block of time and the rest were assigned to the second;

- Scenario 3: All suppliers who came during the period from 8 a.m. to 11 a.m. were assigned to the first block of time and the rest were assigned to the second.

In order to define the properties of the simulation scenarios, we had to vary the parameters listed below:

- the number of supplier arrivals in defined blocks of time - controlled by the mean parameter of probability distribution of supplier arrivals;

- the proportion of the type of delivery with respect to the delivery processing time in defined blocks of time - which was set according to the information obtained from the collected sample about the time of arrival of suppliers and the type of delivery based on the formed clusters.

After we had defined the above-mentioned parameters for each block of time in all three scenarios, we performed a simulation process similar to that used for the simulation of the current state. In each of the four stages of the simulation, each block of time was treated separately; an exception was the last stage where we calculated the total delivery processing time of single iterations for each block separately, but we also calculated the sum of total processing times of the two blocks.

The feasibility of solutions obtained from the simulation models was tested using:

- typical daily load of the observed process - based on the analysis of time study and statistical modelling and the results of the simulation model of the current state. The average number of supplier arrivals per day and the total daily processing time of deliveries obtained through the simulation of the current state were used for comparison with the results of new simulation scenarios. The Kruskal-Wallis H test [37] was used to test the differences between the total delivery processing times of new simulation scenarios and the simulation of the current state, while the pairwise differences were tested by Dunn's test [38,39].

- information about the system constraints - the time capacity of the supplier reception area was compared with the interquartile range of processing times for all simulation scenarios. 
M. Gudlin, M. Hegedić,

H. Cajner, N. Štefanić
Solving Problems of Interruptions and Multitasking in the Pharmacy of a Large Hospital Centre

The simulation was carried out in the $\mathrm{R} 3.4 .2$, program for statistical computing [40]. Graphic representation of the methodology is shown in Figure 3.
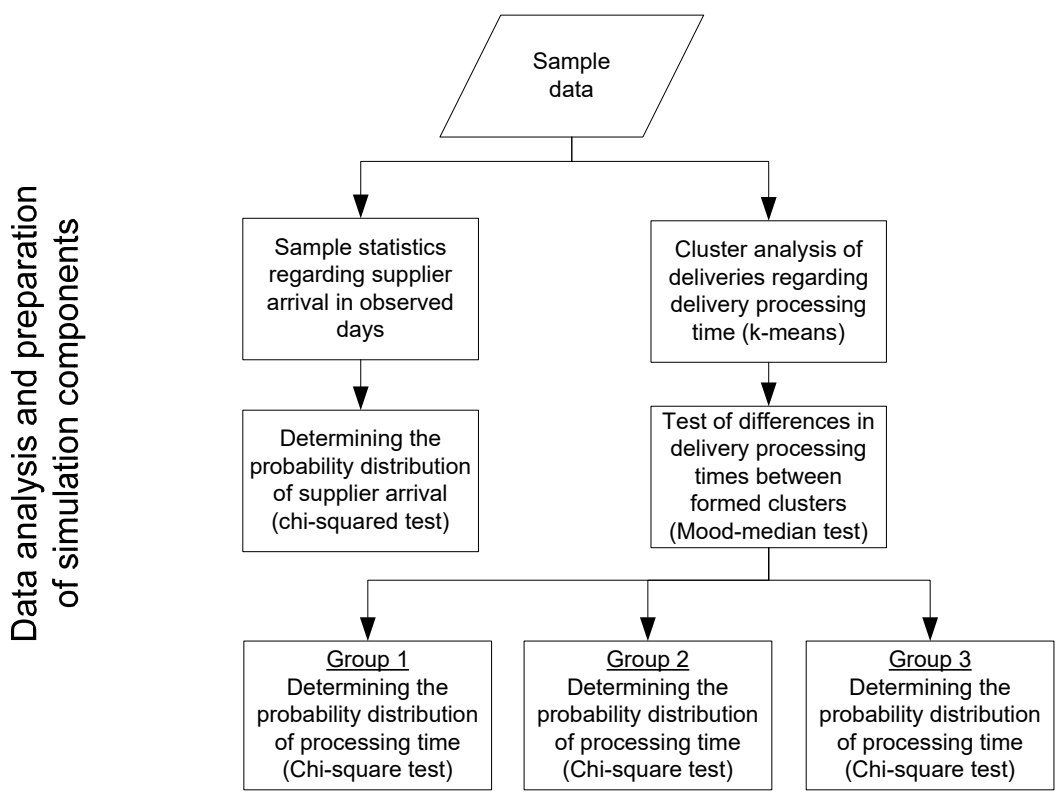

study
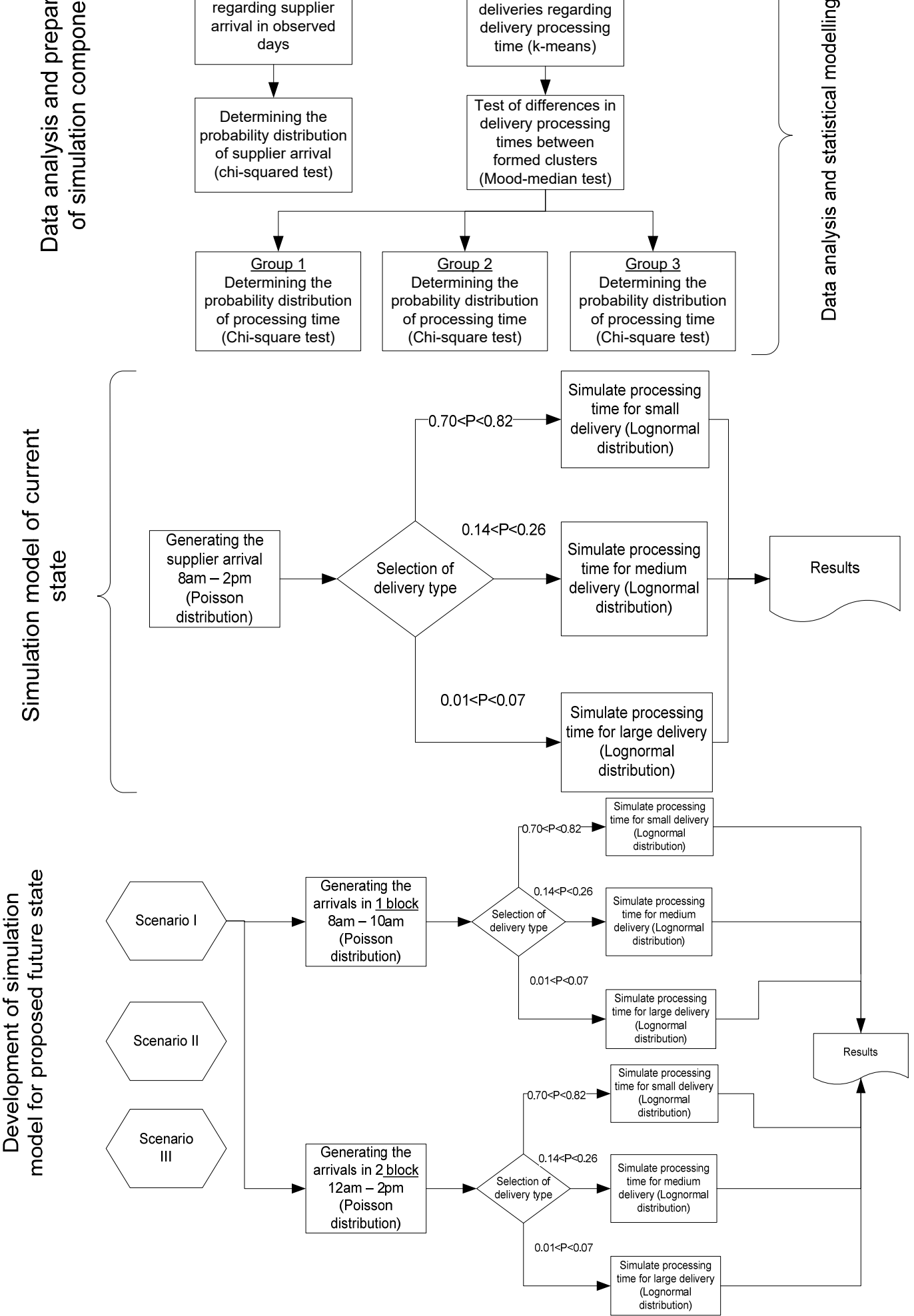

Fig. 3 Simulation methodology 


\section{Results}

\subsection{Time study}

During the time study, 162 deliveries from 48 different suppliers to the pharmacy were recorded. In 119 cases, medical consumables were delivered, 21 delivery contained medications and there were 22 mixed deliveries. The number of arrivals varied between the observed days as shown in Figure 4a, which was modelled as uniform distribution. We also noticed a variation in the number of arrivals between fixed 30-minute periods (Figure $4 \mathrm{~b}$ ), where the most frequent number of deliveries was 2 per 30 minutes.

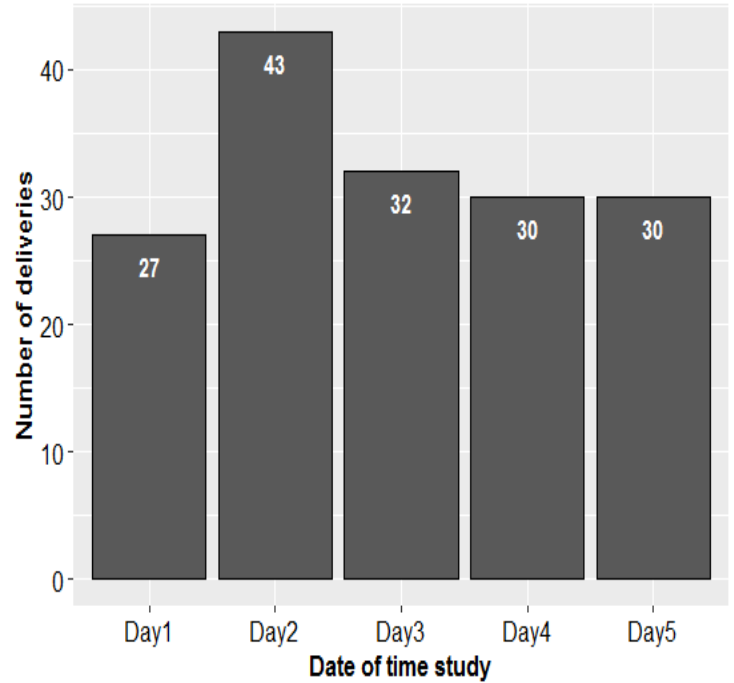

Fig. 4 a) Number of arrivals in the observed days

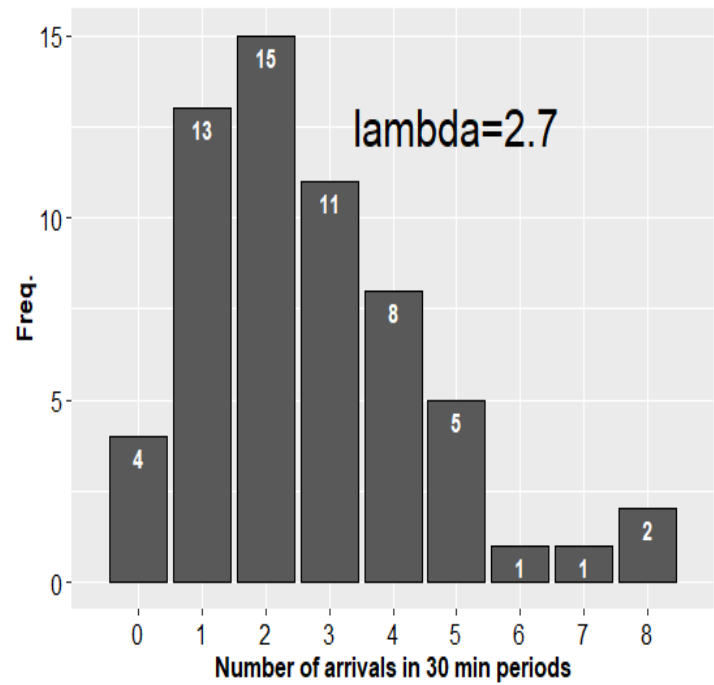

b) Number of arrivals in 30-min periods [33]

A number of supplier arrivals were modelled to follow the Poisson probability distribution (parameter $\lambda=2.7$ ) because the $\mathrm{p}$-value of 0.84 obtained from the $\chi^{2}$ test showed that we could not reject this hypothesis.

\subsection{Data analysis}

The processing time of deliveries, which was an input to the k-means algorithm, gave the most stable results when the centre parameter was set to 3 . The formed delivery groups were divided into small, medium, and large, based on the value of the group centre mean. In the collected sample, there were 123 small deliveries, 33 medium deliveries, and 6 large deliveries, as shown in Figure 5.
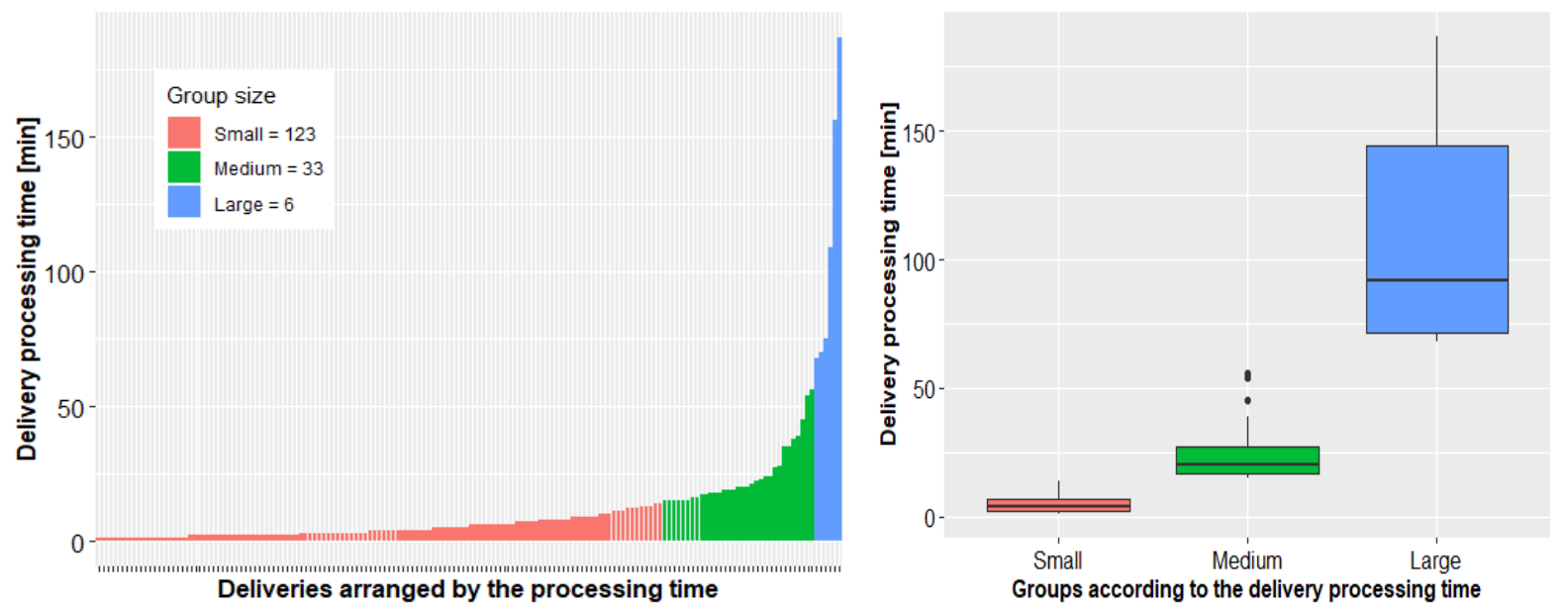

Fig. 5 Types of deliveries according to the processing time [33] 
Mood's median test confirmed that there are significant differences in the processing times between the clustered delivery groups, while the post-hoc tests implied that all the groups are considerably different from each other according to the p-values of pairwise comparisons (small-medium $p$-value $=2.87 \cdot 10^{-13}$, small-large $p$-value $=5.71 \cdot 10^{-3}$, medium-large $p$-value $\left.=8.32 \cdot 10^{-3}\right)$. Statistically significant differences between the groups indicated that deliveries are from different populations, so their processing times needed to be modelled separately. Parameters of group centres (Table 2) obtained by the clustering method were used to estimate the population parameters of processing times for each group.

Table 2 Parameters of group centres [33]

\begin{tabular}{|l|c|c|c|}
\hline Parameter \Group & Small & Medium & Large \\
\hline Mean & 4.715 & 21.433 & 110.833 \\
\hline Std. deviation & 3.474 & 7.065 & 50.261 \\
\hline Range & $1-14$ & $15-56$ & $68-187$ \\
\hline
\end{tabular}

Lognormal distribution was the best fit for the processing time of small deliveries with a p-value of 0.42 obtained in the goodness of fit test. The processing times of medium deliveries were modelled as lognormal because the hypothesis of lognormal distribution could not be rejected in the $\chi^{2}$ test as suggested by the p-value of 0.26 . The goodness of fit test for large deliveries was inconclusive because a small number of deliveries belonging to this group were observed. The decision was to fit large deliveries to the lognormal distribution based on prior experience in the modelling of this type of processes. Population parameters of fitted distributions are shown in Figure 6.
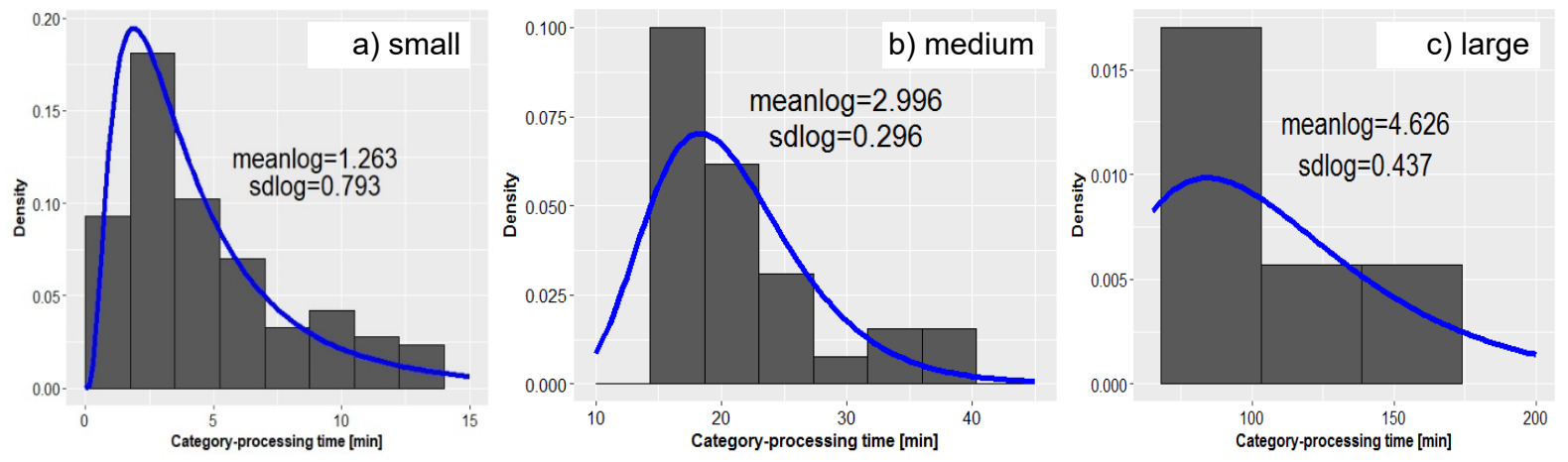

Fig. 6 Fitted distributions of delivery processing times for each group

\subsection{Simulation model of the current state}

The first stage of simulation resulted in 10000 iterations of supplier arrivals (Figure 7) that were generated by the Poisson process with the parameter lambda $=2.7$ and $t=12$ (12 thirty-minute periods in six hours that were observed during a day).

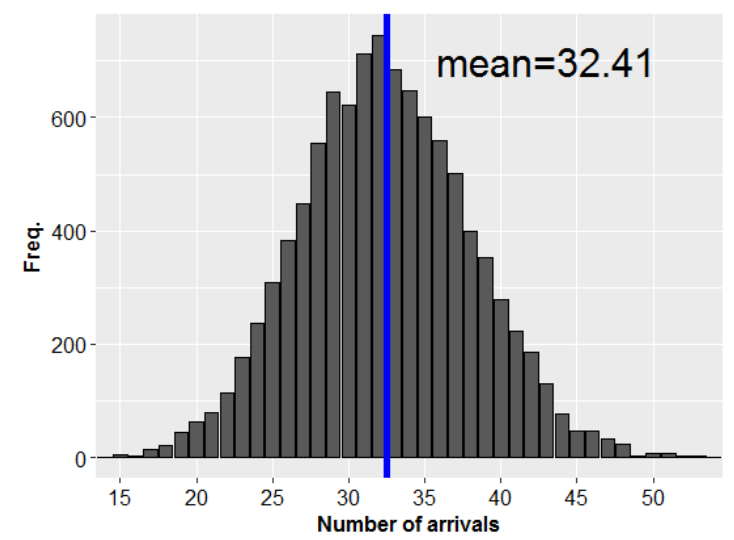

Fig. 7 Distribution of supplier arrivals in the simulation model [33] 
The range of plausible values of supplier arrivals, based on the confidence interval at a level of significance of $5 \%$, calculated from all simulation iterations, was $(22,44)$ arrivals per day.

The next stage connected the delivery types with supplier arrivals through a random number generator, using the proportion of deliveries. In the third stage, randomly generated processing times were added to the matching delivery types from stage 2 . In the final stage, the total processing times of single iterations were calculated and analysed (Figure 8).

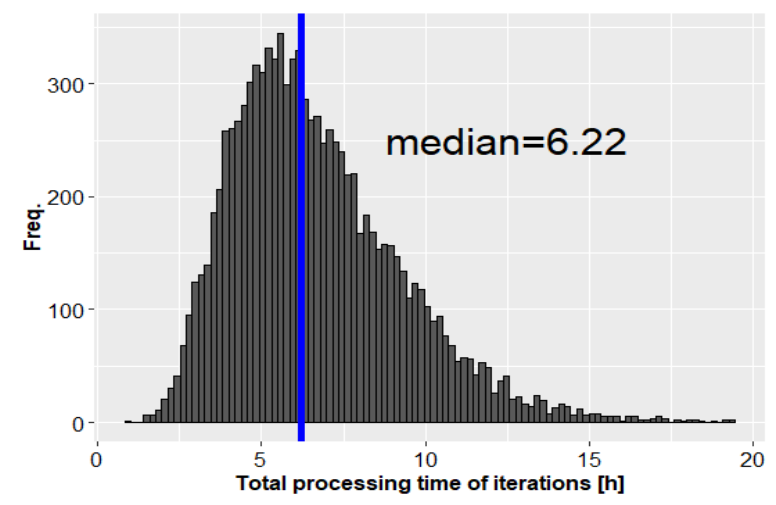

Fig. 8 Distribution of processing times of iterations in the simulation model [33]

The estimated typical daily processing time used for receiving deliveries was 6.22 hours, with an interquartile range of $(4.78,8.16)$ hours based on all simulation iterations 25 th percentile and 75th percentile.

\subsection{Evaluation of the process improvements}

Parameters of each simulation scenario that were varied, namely the average number of supplier arrivals in thirty-minute periods generated by the Poisson distribution $-\lambda 1$ and $\lambda 2$ with $t=4$ (four thirty-minute periods in each block of time), and the proportion of delivery types in the blocks of time are given in Table 3.

Table 3 Parameters varied in the simulation scenarios

\begin{tabular}{|c|c|l|c|l|}
\hline \multirow{2}{*}{$\begin{array}{l}\text { Simulation } \\
\text { scenario }\end{array}$} & \multicolumn{2}{|c|}{ Block of time 1 (8 a.m.-10 a.m.) } & \multicolumn{2}{c|}{ Block of time 2 (12 a.m.-14 a.m.) } \\
\cline { 2 - 5 } & $\lambda_{1}$ & $\begin{array}{l}\text { Proportion of delivery types } \\
\text { (small; medium; large) }\end{array}$ & $\lambda_{2}$ & $\begin{array}{l}\text { Proportion of delivery types } \\
\text { (small, medium, large) }\end{array}$ \\
\hline S1 & 2.20 & $0.730 ; 0.160 ; 0.110$ & 5.90 & $0.770 ; 0.220 ; 0.010$ \\
\hline S2 & 5.25 & $0.800 ; 0.140 ; 0.060$ & 2.85 & $0.680 ; 0.320 ; 0.000$ \\
\hline S3 & 3.90 & $0.808 ; 0.128 ; 0.064$ & 4.21 & $0.714 ; 0.274 ; 0.012$ \\
\hline
\end{tabular}

The distribution of supplier arrivals in block 1 for three simulation scenarios is presented in Figure 9. The heaviest load in the observed pharmacy process related to the average number of supplier arrivals in block 1 was observed in scenario 2, which was expected considering the assumption of scenario 2 regarding the arrival of suppliers in block 1 .

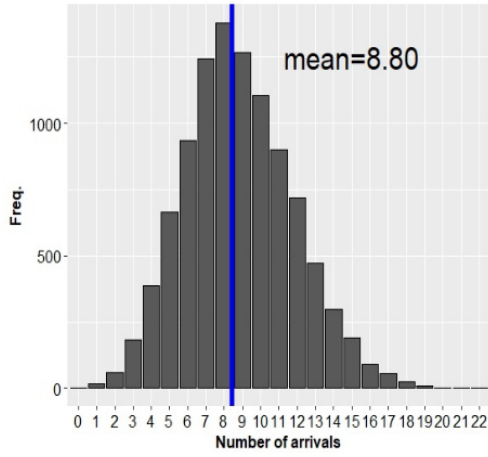

a) scenario 1

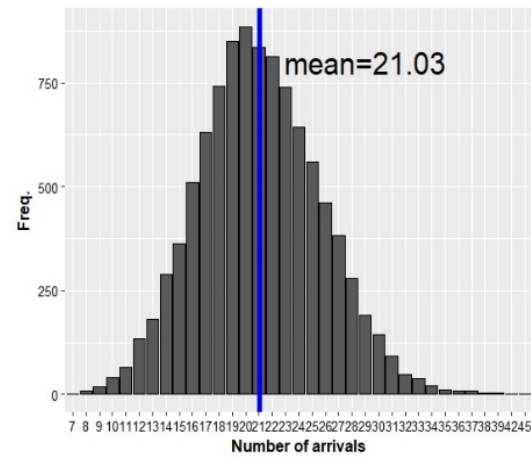

b) scenario 2

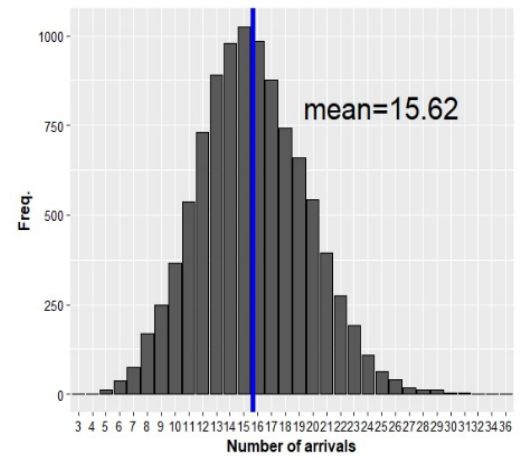

c) scenario 3

Fig. 9 Distribution of supplier arrivals in the block of time 1 for all simulation scenarios 
The distribution of supplier arrivals in block 2 (Figure 10) indicated the largest average number of arrivals in the case of scenario 1 . The biggest difference in the average number of arrivals between block 1 and block 2 was recorded in scenario 1, with $27.2 \%$ of suppliers coming into block 1 and $72.8 \%$ into block 2 on average. Scenario 3 was the most levelled scenario with an average proportion of arrivals in block 1 of up to $48.1 \%$ and up to $51.9 \%$ in block 2 .

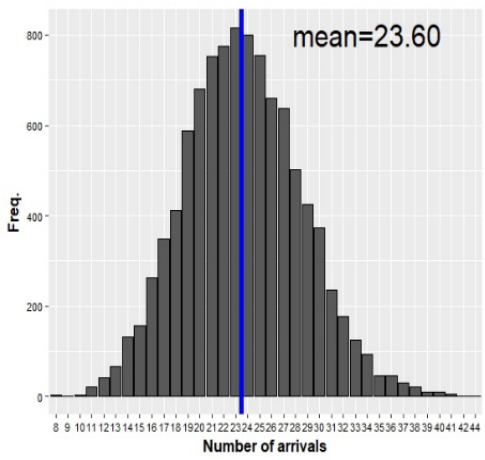

a) scenario 1

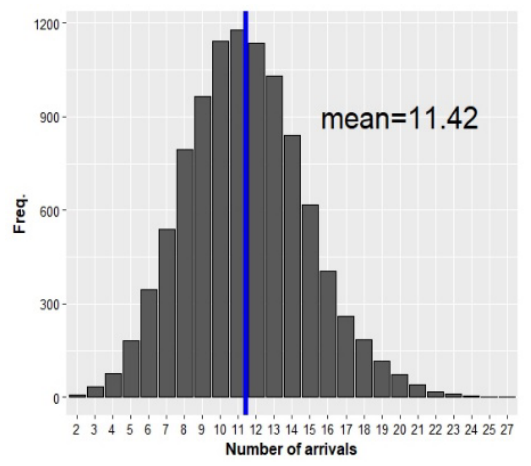

b) scenario 2

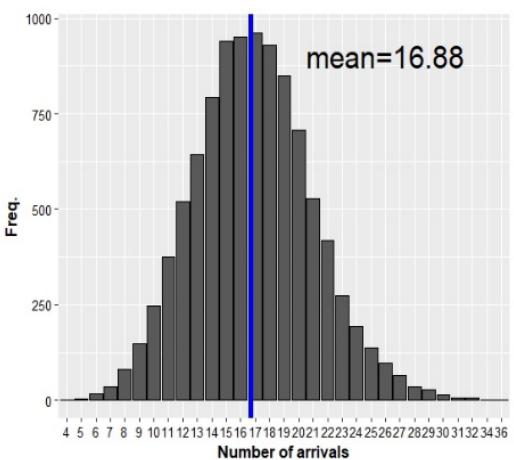

c) scenario 3

Fig. 10 Distribution of supplier arrivals in the block of time 2 for all simulation scenarios

The analysis of the total delivery processing times in block 1 (Figure 11a) indicated that the median of all iterations in scenario 2 is the largest, with 4.30 hours required to receive all the deliveries in that block. In block 2 (Figure 11b), the largest median of the total delivery processing time was recorded in scenario 1 with 3.49 hours.

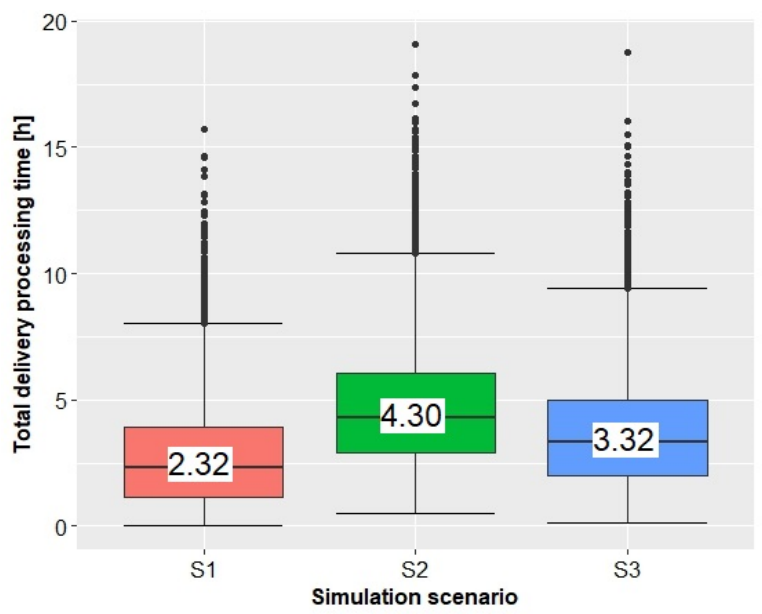

a) block 1

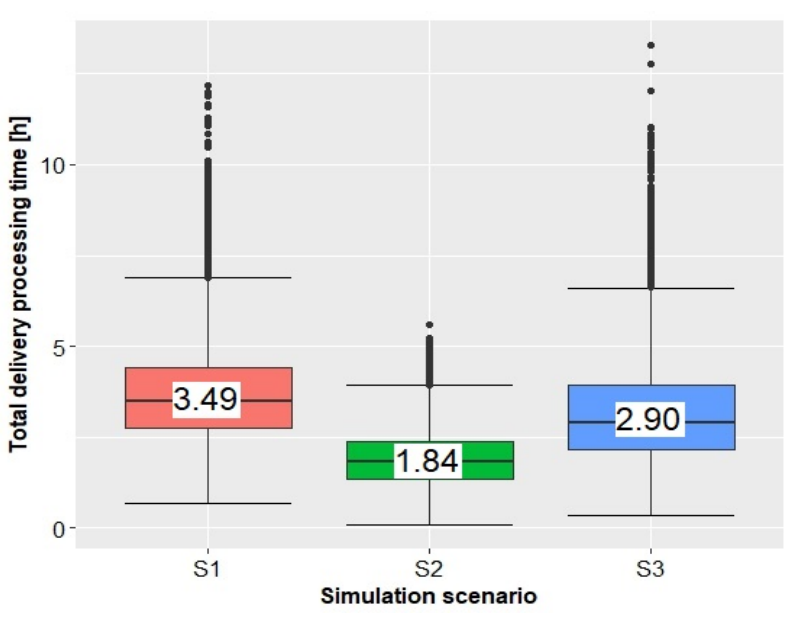

a) block 2

Fig. 11 Distribution of the total delivery processing time for simulation scenarios in block 1 and block 2

The significance of the differences in the distribution of the total delivery processing time between the three simulation scenarios was confirmed by the results of the Kruskal Wallis test for both blocks ( $p$ value $<2.2 \mathrm{e}^{-16}$ ). A comparison of the total daily delivery processing time between the simulation model of the current state and the three simulation scenarios of the new state (Figure 12) was also made using the Kruskal Wallis test. The results of the test showed that there are statistically significant differences in the distribution of the total delivery times across the compared scenarios. Dunn's test of pairwise differences in the distribution of the total delivery processing time of simulation scenarios showed that no significant differences $(\mathrm{p}$ value $=0.18$ ) occurred only between the scenario S0 and the scenario S2. 


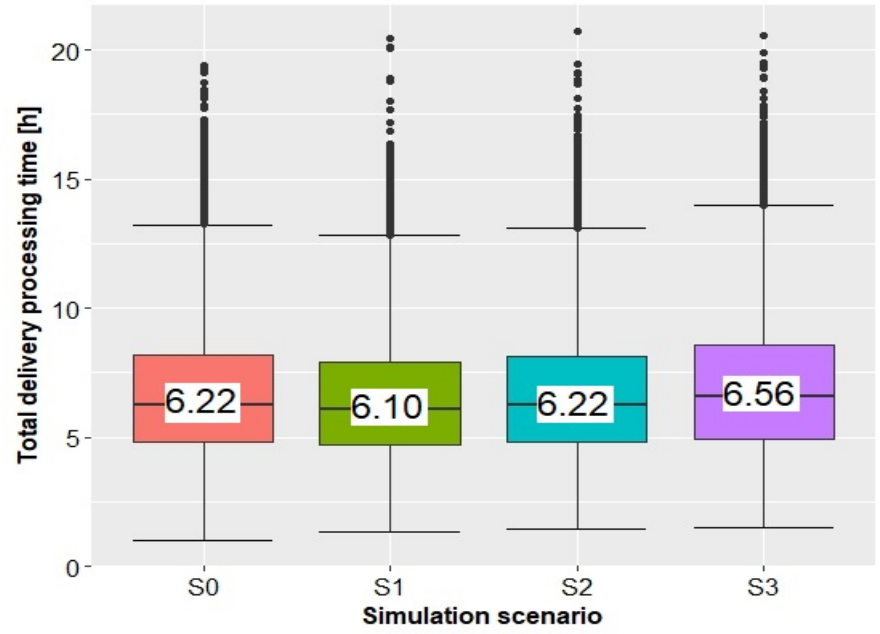

Fig. 12 Distribution of the total delivery processing time for all simulation scenarios including the current state scenario

Information about the interquartile ranges (IQR) of the total delivery processing time for each of the blocks of three simulation scenarios is presented in Table 4. These data were compared to the system constraints, namely the available time of the supplier reception area. Six hours were used as available time of the reception area in each block of time (three reception locations multiplied by two hours available in a block of time).

Table 4 Interquartile ranges of total processing times

\begin{tabular}{|c|c|c|}
\hline \multirow{2}{*}{$\begin{array}{l}\text { Simulation } \\
\text { scenario }\end{array}$} & \multicolumn{2}{|c|}{ Interquartile range of processing time } \\
\cline { 2 - 3 } & Block of time 1 & Block of time 2 \\
\hline S1 & $(1.13,3.90)$ & $(2.76,4.41)$ \\
\hline S2 & $(2.90,6.07)$ & $(1.35,2.38)$ \\
\hline S3 & $(1.99,4.97)$ & $(2.16,3.94)$ \\
\hline
\end{tabular}

The interquartile range exceeds the system constraints only in the case of the first block of time in scenario 2 , which means that more than $25 \%$ of the simulation iterations for scenario 2 in the block 1 resulted in total delivery processing time of over 6 hours.

\section{Discussion}

In this study, our intention was to explore possible improvements in the process of receiving deliveries in a pharmacy, which will lead to a reduction in work-related errors caused by interruptions in work and multitasking. We applied a structured approach to improvements based on statistical modelling and simulations focused on organizational changes in the pharmacy processes. In order to quantify the feasibility of the proposed improvements and to assess the validity of the hypothesis, we have answered the direct research questions put in the introduction of this paper.

Q1: The sample collected during the time study and the subsequent statistical test suggested that the variation in the arrivals of suppliers within the observed days follows the Poisson distribution, which is in agreement with the most common distribution of arrivals in the literature. For example, in papers [21,22], the distribution of patient arrivals is modelled by the Poisson distribution. The reason for modelling the number of supplier arrivals in the observed days with a uniform probability distribution is the fact that the number of hospital patients was more or less unchanged throughout the month and, therefore, there is no 
significant decrease or increase in the demand for pharmacy products. Other possible explanations for approximately the same number of daily deliveries are the effect of the pharmacy's infrastructure (limited stock space) and the defined business policy which does not allow an excessive accumulation of the pharmacy's stock.

Q2: The clustering algorithm and subsequent statistical tests have confirmed that there are significant differences in the delivery processing time between delivery groups. It is interesting to note that in the reviewed literature we found no examples of the application of the clustering method in the pharmacy processes. The largest number of the deliveries belonged to the group of small deliveries. This could be due to the fact that the pharmacy wants to maintain a constant stock level in accordance with the afore-mentioned business policy, so there is a need for frequent small deliveries. The delivery processing time of defined delivery groups corresponded to the lognormal distributions with different parameters, which is comparable to the results obtained in the paper [23], but different from the findings of the authors in the paper [21] where the patient service time (patient processing time) had been modelled by the exponential distribution.

Q3: Based on the results of the S0 simulation model of the current state regarding the average number of supplier arrivals per day and assuming a uniform occupancy of three unloading positions of the reception area, we concluded that a typical daily occupancy of each unloading position is about 135 minutes, which corresponds to 11 deliveries per position. On the assumption that the employees have uniform workload in the delivery admission process and based on the fact that pharmacists are not directly involved in the process of receiving deliveries, our conclusion is that the average daily workload of the employees in this process is approximately 42 minutes (a typical daily delivery processing time of 6.22 divided by 9 employees). Although we gathered information about the type of goods delivered during the time study of the process, we did not use it in the simulation model of the current state. This fact raises additional questions regarding the assumption about a uniform employee workload due to the differences in the average time of receiving deliveries of different types of goods, and this is one of the limitations of this research.

Q4: Examination of the impact of different distributions of the supplier arrivals in defined blocks of time conducted by simulation models showed significant variations in the number of supplier arrivals within the blocks of time between the simulation scenarios S1, S2, and S3. This opens up a possibility of large differences in an employee's daily workload in the defined blocks of time. For example, if the typical events simulated by scenario S1 in block 1 are observed in reality in the first day of observation and the events simulated by scenario S2 in the second day, the difference in the total delivery processing time between the two days could be $87 \%$. If the hospital management decides on a more flexible organization of work of the existing pharmacy employees after the implementation of blocks of time, the aforementioned variability can make it difficult to create daily plans regarding the number of employees required for receiving deliveries. The problem of planning the number of employees in a given day to work on specific pharmacy processes is a possible direction of future research.

Q5: The results of the statistical tests have shown that there are significant differences in the total daily delivery processing time between the model of the current state and three models with defined blocks of time whereas pairwise comparisons have shown that there are no significant differences only between scenarios S0 and S2. The possible interpretation of 
the lack of statistically significant differences between scenarios S0 and S2 is that scenario S2 is the best description of the dynamics of receiving deliveries, as it is in the current state of the pharmacy. Such an interpretation becomes important if we examine the feasibility of the process of receiving deliveries in the case of implemented blocks of time. The feasibility check is based on the total available time of the three unloading positions of the reception area and the interquartile range of the total delivery processing time obtained in the simulation scenarios. Only in the case of block 1 in scenario S2, the feasibility check resulted in the upper limit of the IQR which is greater than the total time capacity; this can be interpreted as a potential risk associated with the implementation of blocks of time. We concluded that the daily activities related to receiving deliveries can be done if blocks of time are used.

\section{Conclusion}

By introducing blocks of time in the process of receiving deliveries, the organization of work in the pharmacy will be changed so that the input and output flows of goods do not overlap. In addition to eliminating the overlapping of flows of goods, the division of work into blocks of time will also reduce interruptions to work and the need for multitasking of employees caused by external factors, such as issuing ordered goods in the middle of the process of receiving a delivery. A prerequisite for such organization of work is a defined policy for emergency issuing of goods, which is necessary due to the nature of work in a hospital. Our assessment, based on the experience and findings of the authors of papers [1015] who researched the effect of multitasking and interruptions on the pharmacy employees and processes, is that such improvements will lead to a decrease in the number of errors in the pharmacy processes. This will result in greater patient satisfaction and a better balance in the employees' work. The final confirmation of the usefulness of the proposed improvement will be made by recording the effect of changes after its implementation in the pharmacy.

An important limitation of the models is the fact that they were developed on a sample of five working days of the same month, which raises the question of model adequacy throughout the year because of a potential impact of seasonality on deliveries (although the pharmacy employees consider the month during which the sample was collected to be the peak period). In addition, it is necessary to examine the impact of changing the process of receiving deliveries on the processes related to it in order to avoid the shifting of the problem to other processes such as issuing of goods. These limitations could be the subject of new research papers.

This paper deals with the improvement in pharmacy processes with a special focus on the process of external delivery and receiving of goods. Since this is an issue which is very rarely explored in literature [30], our study presents an important contribution to the area of research. The proposed approach is based on statistical modelling and simulations. It proved to be an effective solution in the case of a limited amount of data on the current state of the process and of constraints regarding the investment in new pharmacy resources. The approach provides a quick analysis of the impact of potential organizational changes on the hospital pharmacy process and of the possibility for introducing them.

\section{REFERENCES}

[1] R. Pinna, P.P. Carrus, F. Marras, The drug logistics process: an innovative experience, The TQM Journal. 27 (2015) 214-230. https://doi.org/10.1108/TQM-01-2015-0004. 
[2] H. Nachtmann, E.A. Pohl, The state of healthcare logistics: Cost and quality improvement opportunities, Center for Innovation in Healthcare Logistics, University of Arkansas, 2009.

[3] N. Rego, J. Claro, J. Pinho de Sousa, A hybrid approach for integrated healthcare cooperative purchasing and supply chain configuration, Health Care Management Science. 17 (2014) 303-320. https://doi.org/10.1007/s10729-013-9262-y.

[4] J. Beard, M. Ashley, D. Chalkley, Improving the efficiency of a hospital pharmacy service: the journey of one hospital pharmacy, European Journal of Hospital Pharmacy. 21 (2014) 208-215. https://doi.org/10.1136/ejhpharm-2013-000429.

[5] D.T. Barnum, K.L. Shields, S.M. Walton, G.T. Schumock, Improving the Efficiency of Distributive and Clinical Services in Hospital Pharmacy, Journal of Medical Systems. 35 (2011) 59-70. https://doi.org/10.1007/s10916-009-9341-2.

[6] M.S. Alsultan, F. Khurshid, A.Y. Mayet, A.H. Al-jedai, Hospital pharmacy practice in Saudi Arabia: Dispensing and administration in the Riyadh region, Saudi Pharmaceutical Journal. 20 (2012) 307-315. https://doi.org/10.1016/j.jsps.2012.05.003.

[7] C.M. Wittich, C.M. Burkle, W.L. Lanier, Medication Errors: An Overview for Clinicians, Mayo Clinic Proceedings. 89 (2014) 1116-1125. https://doi.org/10.1016/j.mayocp.2014.05.007.

[8] R.N. Keers, S.D. Williams, J. Cooke, D.M. Ashcroft, Causes of Medication Administration Errors in Hospitals: a Systematic Review of Quantitative and Qualitative Evidence, Drug Safety. 36 (2013) 10451067. https://doi.org/10.1007/s40264-013-0090-2.

[9] B. Behzad, R.J. Moraga, S.-J. (Gary) Chen, Modelling healthcare internal service supply chains for the analysis of medication delivery errors and amplification effects, Journal of Industrial Engineering and Management. 4 (2011). https://doi.org/10.3926/jiem.201.

[10] S.P. Desselle, Certified pharmacy technicians' views on their medication preparation errors and educational needs, American Journal of Health-System Pharmacy. 62 (2005) 1992-1997. https://doi.org/10.2146/ajhp040549.

[11] A. Sinclair, D. Terry, M. Slimm, To investigate how disruptive interruptions are on paediatric dispensary accuracy checkers, European Journal of Hospital Pharmacy. 19 (2012) 90.1-90. https://doi.org/10.1136/ejhpharm-2012-000074.15.

[12] T.A. Anacleto, E. Perini, M.B. Rosa, C.C. César, Drug-dispensing errors in the hospital pharmacy, Clinics. 62 (2007) 243-250. https://doi.org/10.1590/S1807-59322007000300007.

[13] V.M. Lea, S.A. Corlett, R.M. Rodgers, Describing interruptions, multi-tasking and task-switching in community pharmacy: a qualitative study in England, International Journal of Clinical Pharmacy. 37 (2015) 1086-1094. https://doi.org/10.1007/s11096-015-0155-7.

[14] E.I. Schafheutle, E.M. Seston, K. Hassell, Factors influencing pharmacist performance: A review of the peer-reviewed literature, Health Policy. 102 (2011) 178-192. https://doi.org/10.1016/j.healthpol.2011.06.004.

[15] C.A. Gaither, Career Commitment: A Mediator of the Effects of Job Stress on Pharmacists' WorkRelated Attitudes, Journal of the American Pharmaceutical Association (1996). 39 (1999) 353-361. https://doi.org/10.1016/S1086-5802(16)30437-5.

[16] K.L. James, D. Barlow, A. Bithell, S. Hiom, S. Lord, M. Pollard, D. Roberts, C. Way, C. Whittlesea, The impact of automation on workload and dispensing errors in a hospital pharmacy: Impact of automation on workload and errors, International Journal of Pharmacy Practice. 21 (2013) 92-104. https://doi.org/10.1111/j.2042-7174.2012.00238.x.

[17] T. Lu, S. Wang, J. Li, P. Lucas, M. Anderson, K. Ross, A Simulation Study to Improve Performance in the Preparation and Delivery of Antineoplastic Medications at a Community Hospital, Journal of Medical Systems. 36 (2012) 3083-3089. https://doi.org/10.1007/s10916-011-9786-y.

[18] O. Hong Choon, C. Wai Leng, W. Jane Ai, T. Mui Chai, Evaluation of manpower scheduling strategies at outpatient pharmacy with discrete-event simulation, OR Insight. 26 (2013) 71-84. https://doi.org/10.1057/ori.2012.9.

[19] F.L. Greenroyd, R. Hayward, A. Price, P. Demian, S. Sharma, Using Evidence-Based Design to Improve Pharmacy Department Efficiency, HERD: Health Environments Research \& Design Journal. 10 (2016) 130-143. https://doi.org/10.1177/1937586716628276. 
[20] T.E. Day, W.M. Li, A. Ingolfsson, N. Ravi, The Use of Queueing and Simulative Analyses to Improve an Overwhelmed Pharmacy Call Center, Journal of Pharmacy Practice. 23 (2010) 492-495. https://doi.org/10.1177/0897190009358772.

[21] M.K. Bahadori, S.M. Mohammadnejhad, R. Ravangard, E. Teymourzadeh, Using Queuing Theory and Simulation Model to Optimize Hospital Pharmacy Performance, Iranian Red Crescent Medical Journal. 16 (2014). https://doi.org/10.5812/ircmj.16807.

[22] C.W. Spry, M.A. Lawley, Evaluating Hospital Pharmacy Staffing and Work Scheduling Using Simulation, in: IEEE, 2005: pp. 2256-2263. https://doi.org/10.1109/WSC.2005.1574514.

[23] M.H. Alhaag, T. Aziz, I.M. Alharkan, A queuing model for health care pharmacy using software Arena, in: IEEE, 2015: pp. 1-11. https://doi.org/10.1109/IEOM.2015.7093849.

[24] B. Sawik, Multi-criteria mathematical programming approaches for assignment of services in hospital, ICIL 2016. (2016) 239À246.

[25] B. Sawik, Weighted-Sum Approach to Health Care Optimization, in: K.D. Lawrence, G. Kleinman (Eds.), Applications of Management Science, Emerald Group Publishing Limited, 2015: pp. 163-180. https://doi.org/10.1108/S0276-897620140000017012.

[26] B. Sawik, A single and triple-objective mathematical programming models for assignment of services in a healthcare institution, International Journal of Logistics Systems and Management. 15 (2013) 249. https://doi.org/10.1504/IJLSM.2013.053770.

[27] A. Jenkins, S.F. Eckel, Analyzing methods for improved management of workflow in an outpatient pharmacy setting, American Journal of Health-System Pharmacy. 69 (2012) 966-971. https://doi.org/10.2146/ajhp110389.

[28] O. Al-Araidah, A. Momani, M. Khasawneh, M. Momani, Lead-Time Reduction Utilizing Lean Tools Applied to Healthcare: The Inpatient Pharmacy at a Local Hospital:, Journal For Healthcare Quality. 32 (2010) 59-66. https://doi.org/10.1111/j.1945-1474.2009.00065.x.

[29] P. Sullivan, S. Soefje, D. Reinhart, C. McGeary, E.D. Cabie, Using lean methodology to improve productivity in a hospital oncology pharmacy, American Journal of Health-System Pharmacy. 71 (2014) 1491-1498. https://doi.org/10.2146/ajhp130436.

[30] J. Volland, A. Fügener, J. Schoenfelder, J.O. Brunner, Material logistics in hospitals: A literature review, Omega. 69 (2017) 82-101. https://doi.org/10.1016/j.omega.2016.08.004.

[31] P. Knudsen, H. Herborg, A.R. Mortensen, M. Knudsen, A. Hellebek, Preventing medication errors in community pharmacy: root-cause analysis of transcription errors, Quality and Safety in Health Care. 16 (2007) 285-290. https://doi.org/10.1136/qshc.2006.022053.

[32] M. Gudlin, M. Hegedić, R. Režek, N. Štefanić, Hospital Pharmacy Layout Optimisation Using Lean Management Principles, in: LEAN Spring Summit 2016 Book of Proceedings, Culmena, Zagreb, Šibenik, Croatia, 2016: pp. 39-56.

[33] M. Gudlin, M. Hegedić, N. Štefanić, H. Cajner, Development of Supplier and Staff Organization Model of Hospital Pharmacy, in: Book of Proceedings of 2nd International Scientific Conference LEAN Spring Summit 2017, Culmena, Zagreb, Šibenik, 2017: pp. 18-26.

[34] T. Kanungo, D.M. Mount, N.S. Netanyahu, C.D. Piatko, R. Silverman, A.Y. Wu, An efficient k-means clustering algorithm: analysis and implementation, IEEE Transactions on Pattern Analysis and Machine Intelligence. 24 (2002) 881-892. https://doi.org/10.1109/TPAMI.2002.1017616.

[35] X. Zhang, J.Z. Huang, D. Qian, J. Xu, L. Jing, Supplier Categorization with K-Means Type Subspace Clustering, in: X. Zhou, J. Li, H.T. Shen, M. Kitsuregawa, Y. Zhang (Eds.), Frontiers of WWW Research and Development - APWeb 2006, Springer Berlin Heidelberg, Berlin, Heidelberg, 2006: pp. 226-237. https://doi.org/10.1007/11610113_21.

[36] C. Robert, G. Casella, Monte Carlo Statistical Methods, Springer-Verlag, New York, 2004.

[37] W.H. Kruskal, W.A. Wallis, Use of Ranks in One-Criterion Variance Analysis, Journal of the American Statistical Association. 47 (1952) 583-621. https://doi.org/10.1080/01621459.1952.10483441.

[38] O.J. Dunn, Multiple Comparisons among Means, Journal of the American Statistical Association. 56 (1961) 52-64. https://doi.org/10.1080/01621459.1961.10482090.

[39] O.J. Dunn, Multiple Comparisons Using Rank Sums, Technometrics. 6 (1964) 241-252.

https://doi.org/10.1080/00401706.1964.10490181. 
M. Gudlin, M. Hegedić,

H. Cajner, N. Štefanić
Solving Problems of Interruptions and Multitasking in the Pharmacy of a Large Hospital Centre

[40] R Core Team, R: A language and environment for statistical computing, R Foundation for Statistical Computing, Vienna, Austria, 2017. https://www.R-project.org/.

Submitted: $\quad$ 20.7.2018

Accepted: $\quad$ 13.3.2019
Mihael Gudlin,

mihael.gudlin@fsb.hr

Dr.sc. Miro Hegedić,

miro.hegedic@fsb.hr

Doc. dr. sc. Hrvoje Cajner,

hrvoje.cajner@fsb.hr

Prof. dr. sc. Nedeljko Štefanić

nstefan@fsb.hr

Faculty of Mechanical Engineering and

Naval Architecture,

Ivana Lučića 5, 10002 Zagreb, Croatia 